\title{
Effect of Levofloxacin and Vitamin C on Bacterial Adherence and Preformed Biofilm on Urethral Catheter Surfaces
}

\author{
Eman El-Gebaly1, Tamer Essam²*, Shabaan Hashem ${ }^{3}$ and Rehab A. El-Baky ${ }^{4}$ \\ ${ }^{1}$ Microbiology and Immunology Department, Faculty of Pharmacy, Beni Seuif University, Egypt \\ ${ }^{2}$ Microbiology and Immunology Department and Biotechnology Centre, Faculty of Pharmacy, Cairo University, Kasr El-Aini Street, Cairo11562, Egypt \\ ${ }^{3}$ Microbiology and Immunology Department, Faculty of Medicine, Assuit University, Egypt \\ ${ }^{4}$ Microbiology and Immunology Department, Faculty of Pharmacy, Minia University, Egypt
}

\begin{abstract}
The effect of levofloxacin (LEV) and vitamin-C (VIT-C) individually or in combinations on initial bacterial adherence and pre-formed (mature) biofilms on the surface of urethral catheters was studied. The isolated and studied uropathogens in the present study showed considerable diversity, where the major pathogens were identified as E.coli, Klebsiella sp., Citrobacter sp., Enterobacter sp., Proteus $s p$. and Pseudomonas sp. Using the static adherence assay, addition of LEV and VIT-C each alone at sub MIC concentrations ( $0.25 \mathrm{MIC}$ and $0.5 \mathrm{MIC})$ and $(80$ and $100 \mathrm{mg} \mathrm{ml}-1$ ) respectively, reduced the initial adherence ability of bacteria to the catheter by $35-94 \%$. Besides, the inhibitory effect on the mature biofilm was estimated to be $40-90 \%$ in the presence of (MIC and 2MIC) LEV or ( 80 and $100 \mathrm{mg} / \mathrm{ml}$ ) VIT-C. The highest inhibitory effects on both the initial adherence and mature biofilm were recorded when $0.5 \mathrm{MIC}$ levofloxacin was added. Combination of LEV and VIT-C significantly increased the inhibitory effect of both initial biofilm formation and the mature biofilm to $80-100 \%$. In addition, scanning electron microscope (SEM) was used to verify the effect of the tested drugs on biofilm production. The obtained results confirmed the significant role of this combination in inhibition of urethral catheter biofilm formation.
\end{abstract}

Keywords: Ascorbic acid (vitamin-C); Biofilm; Fluoroquinolones; Levofloxacin; Urinary tract

\section{Introduction}

Catheter associated urinary tract infection (CAUTI) is one of the most common types of hospital acquired infection [1,2]. Several studies have confirmed its significant contribution in increasing the morbidity, mortality, hospital stay and costs [3]. The normal function of the urinary tract is usually altered by the presence of a catheter and this makes it easier for bacteria to become established in the bladder and cause serious infection [1]. A wide range of persistent catheter related infections may be related to the ability of bacteria to form biofilms [4,5]. In this regards, the treatment of device related infections with conventional antimicrobial agents frequently fails because microorganisms growing in biofilms are more tolerant or phenotypically resistant to antimicrobial agents than planktonic (free) cells [4]. A mature biofilm can even tolerate antibiotics at concentrations of 10-1000 times more than required to kill planktonic bacteria [6]. In this regard, fluoroquinolones (The most common clinically used members of the quinolones antibiotics) have been reported as an effective treatment in case of both young and mature biofilms because of their good penetrative qualities [7]. Norrby et al. [8] have reported that among the recently developed fluoroquinolones, levofloxacin is widely used in clinical practice and it is less likely to select resistant strains compared with other group members. Therefore, levofloxacin represent a promising candidate to treat tedious infections such as those of biofilm forming bacteria [9].

However, the use of levofloxacin alone may jeopardous its high activity to fast development of resistance. Fortunately, several reports have stated that the antibacterial effect of levofloxacin was enhanced by ascorbic acid (VIT-C) [10]. Interestingly, Habash et al. [11] have reported that the use of ascorbic acid (VIT-C) provided a degree of protection against adhesion by some uropathogens and colonization of biomaterials utilized within the urinary tract. Therefore, it is conceivable that an antibiofilm/antimicrobial agent combination would be synergistic [12].
In this prospective, this study investigated the adherence capacity of common isolated uropathogens on urinary catheters. The effect of levofloxacin and vitamin- $\mathrm{C}$ either individually or in combinations on the adherence ability as well as the mature formed biofilms by uropathogens on urinary catheters was evaluated.

\section{Materials and Methods}

Unless otherwise specified, all tests were conducted under aseptic conditions and in triplicates and the presented data is mean \pm standard error.

\section{Uropathogenes}

Previously, uropathogenic bacterial isolates were collected from catheters and stents from 115 inpatients at Beni Seuif University hospital. All isolated uropathogenes in the present study were subjected to systematic identification based on morphological characteristics and biochemical reactions using API Test Kits (20E and 20NE API, bioMerieux, France; data not shown). The antibiogram and minimum inhibitory concentration (MIC) for all isolates were determined (data not shown). Among the isolated and preliminary identified bacterial uropathogens, 2 isolates of each common species (E.coli, Klebsiella sp., Citrobacter sp., Enterobacter sp., Proteus sp. and Pseudomonas sp.) were selected to be used in this study. Selection was based on the pre-

\footnotetext{
*Corresponding author: Tamer Essam, Microbiology and Immunology Department and Biotechnology Centre, Faculty of Pharmacy, Cairo University, Kasr El-Aini Street, Cairo11562, Egypt, E-mail: tamer.essam@yahoo.com

Received October 02, 2012; Accepted October 25, 2012; Published October 29 , 2012

Citation: El-Gebaly E, Essam T, Hashem S, El-Baky RA (2012)Effect of Levofloxacin and Vitamin C on Bacterial Adherence and Preformed Biofilm on Urethral Catheter Surfaces. J Microb Biochem Technol 4: 131-136. doi:10.4172/1948-5948.1000083

Copyright: (C) 2012 El-Gebaly E, et al. This is an open-access article distributed under the terms of the Creative Commons Attribution License, which permits unrestricted use, distribution, and reproduction in any medium, provided the original author and source are credited
} 
determined MIC in order to have one with the highest MIC and the other with lowest MIC (Table 1). All isolates were stored on slants of corresponding culture media at $4^{\circ} \mathrm{C}$ according to [13]

\section{Drugs}

Stock solutions of levofloxacin $(5000 \mu \mathrm{g} / \mathrm{ml})$ and Vitamin-C (1000 $\mathrm{mg} / \mathrm{ml}$ ) were prepared in sterile distilled water. Both drugs were purchased from Sedico, Egypt and stored according to manufacturer instructions.

\section{Determination of minimum inhibitory concentrations (MIC) of levofloxacin}

Minimum inhibitory concentrations of levofloxacin were determined using the microbroth dilution method according to Clinical Laboratory Standard Institute (CLSI 2009) [14].

Effect of levofloxacin and vitamin-C (alone and in combination) on initial biofilm formation and on the preformed biofilm

Bacterial cultures in $5 \mathrm{ml}$ tryptic soy broth (TSB) of the tested uropathogens were diluted 100 times with fresh TSB and distributed as potions of $5 \mathrm{ml}$ into test tubes. One of the following solutions: LEV (0.25 MIC and 0.5 MIC), VIT-C ( 80 and $100 \mathrm{mg} / \mathrm{ml})$ and LEV/ VIT-C $(0.25 \mathrm{MIC} / 80 \mathrm{mg} / \mathrm{ml}$ and $0.5 \mathrm{MIC} / 100 \mathrm{mg} / \mathrm{ml})$ were added to each tube as triplicates. One set of seven pieces of urethral catheters segments (each of $1 \mathrm{~cm}$ length) was added to each tube and all tubes were statically incubated at $37^{\circ} \mathrm{C}$ for $24 \mathrm{~h}$. After incubation, catheter segments were rinsed 3 times with phosphate buffer saline (PBS, $\mathrm{pH}$ 7.2), placed in $10 \mathrm{ml}$ fresh saline and sonicated for 30 seconds to dislodge the adherent cells. The sonicated saline was serially diluted and cultured on tryptic soy agar plates and the number of adherent bacteria that indicates degree of adherence was determined by the viable count technique [15]. Control was conducted by addition of normal saline and treated in the same regimen, with the addition of saline in lieu of TSB. The effect of the tested agents on the pre-formed (mature) biofilm was tested by incubating seven pieces of the $1 \mathrm{~cm}$ urethral catheter segments in test tubes containing the selected bacterial suspension and $5 \mathrm{ml}$ of TSB to allow biofilm formation. After incubation at $37^{\circ} \mathrm{C}$ for $24 \mathrm{~h}$., segments were removed and rinsed 3 times with (PBS, $\mathrm{pH}$ 7.2) to remove any non-adherent bacteria. Catheter segments were then suspended for $24 \mathrm{~h}$ at $37^{\circ} \mathrm{C}$ in one of the following treatment solutions: saline (control), levofloxacin (MIC and 2 MIC), vitamin-C (80 and $100 \mathrm{mg} / \mathrm{ml}$ ) and LEV/VIT-C (MIC/80 and $2 \mathrm{MIC} / 100 \mathrm{mg} / \mathrm{ml})$. After incubation, the number of viable adherent cells was determined as previously described.

\section{Scanning Electron Microscopy (SEM)}

Catheter segments were fixed in $2.5 \%(\mathrm{v} / \mathrm{v})$ glutaraldhyde in PBS ( $\mathrm{pH}$ 7.2) for $1.5 \mathrm{~h}$, rinsed with PBS and then dehydrated through an ethanol series. Samples were dried and gold-palladium coated. SEM examinations were made on a JSM-840 SEM (JEOL Ltd., Tokyo, Japan) [13].

\section{Statistical analysis}

One-way ANOVA was employed to evaluate any significant difference between the values obtained without the drug (controls) and the values obtained in the presence of different drug concentrations. Differences were done using INSTAT statistical software (GraphPad Instant V 2.03, GraphPad Software Inc., USA).

\begin{tabular}{|c|c|c|}
\hline Species & $\begin{array}{c}\text { Isolate } \\
\text { No. }\end{array}$ & MIC of levofloxacin $\left(\mu \mathrm{g} \mathrm{ml}^{-1}\right)$ \\
\hline \multirow{2}{*}{ E. coli } & 27 & 128 \\
\hline & 98 & 4 \\
\hline \multirow{2}{*}{ Klebsiella sp. } & 56 & 4 \\
\hline & 58 & 2 \\
\hline \multirow{2}{*}{ Citrobacter $s p$} & 22 & 2 \\
\hline & 77 & 64 \\
\hline \multirow{2}{*}{ Enterobacter sp. } & 29 & 64 \\
\hline & 94 & 2 \\
\hline \multirow{2}{*}{ Proteus sp. } & 102 & 16 \\
\hline & 131 & 128 \\
\hline \multirow{2}{*}{ Pseudomonas sp. } & 86 & 2 \\
\hline & 133 & 16 \\
\hline
\end{tabular}

\section{Results}

\section{Effect of levofloxacin and/or vitamin-C on biofilm production}

Levofloxacin at $0.25 \mathrm{MIC}$ inhibited the initial adherence of all tested bacterial uropathogens. The recorded inhibition was widely ranged from 40-77\% (Table 2). This inhibitory effect was increased with different extents when the concentration increased to 0.5 MIC. Similarly, VIT-C at $80 \mathrm{mg} / \mathrm{ml}$ showed also an inhibitory effect ranging from $60-80 \%$ on all tested bacterial uropathogens. Again this inhibitory effect was slightly increased by increasing VIT-C concentration to $100 \mathrm{mg} / \mathrm{ml}$. Combination between $0.25 \mathrm{MIC}$ of (LEV) and $80 \mathrm{mg} / \mathrm{ml}$ of (VIT-C) inhibited the bacterial adherence of all tested isolates with almost the same range as VIT-C alone. However, when the concentration increased to $0.5 \mathrm{MIC}$ and $100 \mathrm{mg} / \mathrm{ml}$, the recorded inhibitory effect was always above $90 \%$. In most cases, upon combination of LEV and VIT-C $(0.25 \mathrm{MIC} / 80 \mathrm{mg} / \mathrm{ml})$, the inhibitory effect on the biofilm formation ability was increased (Table 2). The highest inhibitory effect was observed on Proteus sp.131 while the lowest inhibitory effect (60.7\%) was observed on Enterobacter sp. 94. When the concentration of LEV/VIT-C combination was increased to $0.5 \mathrm{MIC} / 100 \mathrm{mg} / \mathrm{ml}$, the inhibition was always above $90 \%$ and in 2 cases complete inhibition was recorded. Interestingly, the same pattern of inhibitory effect was always consistent regardless the sensitivity or resistance of the tested uropathogens. For instance, the highest inhibitory effect on E. coli 27 (most resistant isolate) and Pseudomonas sp. 86 (most sensitive isolate) was recorded when a combination of $0.5 \mathrm{MIC} / 100 \mathrm{mg} / \mathrm{ml}$ VIT-C was used (Figure 1). Again, the lowest inhibitory effect against both bacteria was recorded when 0.25 MIC LEV was used alone.

\section{Effect of levofloxacin and vitamin-C on the pre-formed (mature) biofilm}

No significant inhibitory effect was recorded on all tested uropathogens when 0.25 and $0.5 \mathrm{MIC}$ concentrations were tested (data not shown); therefore, levofloxacin was tested at higher concentrations (MIC and 2 MIC). Application of LEV at MIC inhibited the mature biofilm by a range of $20-65 \%$ (Table 3 ). Increasing this concentration to 2 MIC increased the inhibitory effect to different extents (up to 91\%). Similarly, VIT-C at $80 \mathrm{mg} / \mathrm{ml}$ showed a wide range of inhibition (27-79\%). The increase in VIT-C concentration to $100 \mathrm{mg} / \mathrm{ml}$ was accompanied with an increase in the inhibitory effect (Table 3). Combination between LEV and VIT-C at MIC/80 mg/ml showed almost similar inhibitory effect to that recorded for VIT-C alone (33$82 \%)$. The highest inhibitory effects (83-99.6\%) were recorded when a combination of $2 \mathrm{MIC} / 100$ was used (Table 3 ). 
Citation: El-Gebaly E, Essam T, Hashem S, El-Baky RA (2012) Effect of Levofloxacin and Vitamin C on Bacterial Adherence and Preformed Biofilm on Urethral Catheter Surfaces. J Microb Biochem Technol 4: 131-136. doi:10.4172/1948-5948.1000083

\begin{tabular}{|c|c|c|c|c|c|c|c|c|c|}
\hline \multirow[b]{2}{*}{ Uropathogen } & \multicolumn{3}{|c|}{ Levofloxacin } & \multicolumn{3}{|c|}{ Vitamin-C } & \multicolumn{3}{|c|}{ Levofloxacin/Vitamin-C } \\
\hline & $\begin{array}{l}\text { Conc. } \\
\mu \mathrm{g} / \mathrm{ml}\end{array}$ & $\begin{array}{c}\text { V.C } \\
\text { cfu×1033 } \\
\pm \text { SEM }\end{array}$ & Reduction \% & $\begin{array}{l}\text { Conc. } \\
\mathrm{mg} / \mathrm{ml}\end{array}$ & $\begin{array}{c}\text { V.C } \\
\text { cfu× } 10^{3} \\
\pm \text { SEM }\end{array}$ & Reduction\% & $\begin{array}{l}\text { Conc. } \\
(\mu \mathrm{g} / \mathrm{ml}) /(\mathrm{mg} / \\
\mathrm{ml})\end{array}$ & $\begin{array}{c}\text { VC } \\
\text { cfu×1033 } \\
\pm \text { SEM }\end{array}$ & Reduction \% \\
\hline \multirow{3}{*}{$\begin{array}{l}\text { E. coli } \\
(27)\end{array}$} & CTR & $160 \pm 5$ & & & $160 \pm 5$ & & & $160 \pm 5$ & \\
\hline & $32^{\mathrm{a}}$ & $56 \pm 0.6^{*}$ & 65 & 80 & $48 \pm 1.4^{*}$ & 70 & $32 / 80^{\circ}$ & $40 \pm 0.68^{*}$ & 75 \\
\hline & $64^{\mathrm{b}}$ & $24 \pm 0.64^{*}$ & 85 & 100 & $20 \pm 0.76^{*}$ & 87.5 & $64 / 100^{d}$ & $0.5 \pm 0.07^{*}$ & 99.7 \\
\hline \multirow{3}{*}{ (98) } & CTR & $40 \pm 0.86$ & & & $40 \pm 0.86$ & & & $40 \pm 0.86$ & \\
\hline & $1^{\mathrm{a}}$ & $12 \pm 0.56^{*}$ & 70 & 80 & $8 \pm 0.6^{*}$ & 80 & $1 / 80^{c}$ & $10 \pm 0.8^{*}$ & 75 \\
\hline & $2^{b}$ & $1.4 \pm 0.17^{*}$ & 96.5 & 100 & $3.2 \pm 0.4^{*}$ & 92 & $2 / 100^{d}$ & $0.3 \pm 0.05^{*}$ & 97.25 \\
\hline \multirow{3}{*}{ Klebsiella sp. (56) } & CTR & $56 \pm 0.64$ & & & $56 \pm 0.64$ & & & $56 \pm 0.64$ & \\
\hline & $1^{\mathrm{a}}$ & $34 \pm 0.6^{*}$ & 40 & 80 & $14 \pm 0.97^{*}$ & 60 & $1 / 80^{c}$ & $21 \pm 0.97^{*}$ & 75 \\
\hline & $2^{b}$ & $19 \pm 1^{*}$ & 65 & 100 & $8.4 \pm 0.75^{*}$ & 85 & $2 / 100^{d}$ & $0.1 \pm 0.02^{*}$ & 99.8 \\
\hline \multirow{3}{*}{$(58)$} & CTR & $25 \pm 1$ & & & $25 \pm 1$ & & & $25 \pm 1$ & \\
\hline & $0.5^{\mathrm{a}}$ & $13 \pm 1.1^{*}$ & 50 & 80 & $6.2 \pm 0.7^{*}$ & 75 & $0.5 / 80^{c}$ & $5.7 \pm 0.4^{*}$ & 77 \\
\hline & $1^{\mathrm{b}}$ & $7.5 \pm 0.56^{*}$ & 70 & 100 & $3.8 \pm 0.4^{*}$ & 85 & $1 / 100^{d}$ & $0.45 \pm 0.02^{*}$ & 90 \\
\hline & & & & & & & & & \\
\hline \multirow{3}{*}{ Citrobacter sp. (22) } & CTR & $170 \pm 10.4$ & & & $170 \pm 10.4$ & & & $170 \pm 10.4$ & \\
\hline & $0.5^{\mathrm{a}}$ & $58.8 \pm 1.6^{*}$ & 65 & 80 & $68 \pm 1.3^{*}$ & 60 & $0.5 / 80^{c}$ & $51 \pm 1.6^{*}$ & 70 \\
\hline & $1^{b}$ & $8.8 \pm 0.7^{*}$ & 94.8 & 100 & $42 \pm 1.6^{*}$ & 75 & $1 / 100^{d}$ & $1.7 \pm 0.25^{*}$ & 99 \\
\hline \multirow{3}{*}{ (77) } & CTR & $120 \pm 0.57$ & & & $120 \pm 0.57$ & & & $120 \pm 0.57$ & \\
\hline & $16^{a}$ & $54 \pm 0.76^{*}$ & 55 & 80 & $36 \pm 1.3^{*}$ & 70 & $16 / 80^{c}$ & $30 \pm 1.3^{*}$ & 75 \\
\hline & $32^{b}$ & $18 \pm 1^{*}$ & 85 & 100 & $13 \pm 1.1^{*}$ & 89 & $32 / 100^{d}$ & $9 \pm 0.8^{*}$ & 92 \\
\hline & & & & & & & & & \\
\hline \multirow{3}{*}{ Enterobacter sp. (29) } & CTR & $140 \pm 5.7$ & & & $140 \pm 5.7$ & & & $140 \pm 5.7$ & \\
\hline & $16^{a}$ & $70 \pm 5^{*}$ & 50 & 80 & $35 \pm 1^{*}$ & 75 & $16 / 80^{c}$ & $32 \pm 1.1^{*}$ & 77 \\
\hline & $32^{b}$ & $15 \pm 0.76^{*}$ & 89 & 100 & $14 \pm 1^{*}$ & 90 & $32 / 100^{d}$ & 0 & 100 \\
\hline \multirow{3}{*}{ (94) } & CTR & $56 \pm 0.76$ & & & $56 \pm 0.76$ & & & $56 \pm 0.76$ & \\
\hline & $0.5^{\mathrm{a}}$ & $32 \pm 1^{*}$ & 43 & 80 & $12 \pm 0.49^{*}$ & 78.6 & $0.5 / 80^{c}$ & $22 \pm 0.68^{\star}$ & 60.7 \\
\hline & $1^{\mathrm{b}}$ & $4 \pm 0.4^{*}$ & 93 & 100 & $8.4 \pm 0.3^{*}$ & 85 & $1 / 100^{d}$ & $0.5 \pm 0.05^{*}$ & 99 \\
\hline & & & & & & & & & \\
\hline \multirow{3}{*}{$\begin{array}{l}\text { Proteus sp. } \\
(102)\end{array}$} & CTR & $140 \pm 5.7$ & & & $140 \pm 5.7$ & & & $140 \pm 5.7$ & \\
\hline & $4^{\mathrm{a}}$ & $42 \pm 1.5^{*}$ & 70 & 80 & $49 \pm 1.3^{*}$ & 65 & $4 / 80^{c}$ & $32.2 \pm 1.1^{*}$ & 77 \\
\hline & $8^{\mathrm{b}}$ & $21 \pm 1^{*}$ & 85 & 100 & $21 \pm 0.76^{*}$ & 85 & $8 / 100^{d}$ & $2.8 \pm 0.3^{*}$ & 98 \\
\hline \multirow{3}{*}{ (131) } & CTR & $60 \pm 1$ & & & $60 \pm 1$ & & & $60 \pm 1$ & \\
\hline & $32^{\mathrm{a}}$ & $14 \pm 0.4^{*}$ & 77 & 80 & $13 \pm 0.55^{*}$ & 78 & $32 / 80^{c}$ & $9 \pm 0.5^{*}$ & 85 \\
\hline & $64^{\mathrm{b}}$ & $11 \pm 0.5^{*}$ & 82 & 100 & $5.4 \pm 0.3^{*}$ & 91 & $64 / 100^{d}$ & 0 & 100 \\
\hline & & & & & & & & & \\
\hline \multirow{3}{*}{$\begin{array}{l}\text { Pseudomonas } \\
\text { (86) }\end{array}$} & CTR & $65 \pm 1.4$ & & & $65 \pm 1.4$ & & & $65 \pm 1.4$ & \\
\hline & $0.5^{\mathrm{a}}$ & $32.5 \pm 1.2^{*}$ & 45 & 80 & $29 \pm 1.5^{\star}$ & 70 & $0.5 / 80^{c}$ & $19.5 \pm 1.2^{*}$ & 80 \\
\hline & $1^{\mathrm{b}}$ & $19.5 \pm 1.8^{*}$ & 85 & 100 & $1.9 \pm 0.3^{*}$ & 83 & $1 / 100^{d}$ & $5.8 \pm 0.44^{*}$ & 99.9 \\
\hline \multirow{3}{*}{ (133) } & CTR & $68 \pm 1.5$ & & & $68 \pm 1.5$ & & & $68 \pm 1.5$ & \\
\hline & $4^{a}$ & $41 \pm 1.1^{*}$ & 40 & 80 & $16 \pm 0.5^{*}$ & 77 & $4 / 80^{\circ}$ & $14 \pm 1^{*}$ & 80 \\
\hline & $8^{\mathrm{b}}$ & $6 \pm 0.52^{*}$ & 75 & 100 & $7.5 \pm 0.3^{*}$ & 89 & $8 / 100^{d}$ & $4 \pm 0.7^{\star}$ & 94 \\
\hline
\end{tabular}

a) The used concentration was equal to $0.25 \mathrm{MIC}$ of levofloxacin alone.

b) The used concentration was equal to $0.5 \mathrm{MIC}$ of levofloxacin alone.

c) The used concentration was equal to $0.25 \mathrm{MIC}$ of levofloxacin and $80 \mathrm{mg}$ vitamin $\mathrm{C} / \mathrm{ml}$.

d) The used concentration was equal to $0.5 \mathrm{MIC}$ of levofloxacin and $100 \mathrm{mg}$ vitamin $\mathrm{C} / \mathrm{ml}$.

(V.C) means the viable cell counts on the surface of urethral catheters.

(CTR) means the control without drug.

${ }^{*} \mathrm{P}<0.05$ : Significant value, compared to controls.

Table 2: Effects of levofloxacin, vitamin-C alone and in combination on initial adherence of the tested uropathogens on the catheter surfaces

The highest inhibitory effect of LEV at MIC was observed on Proteus sp 102 (65\%) while the lowest inhibitory effect (20\%) was recorded on Klebsiella sp. 58. When LEV was used at 2 MIC, the highest inhibitory effect (91\%) was recorded against Pseudomonas sp. 133. Again Klebsiella sp. 58 was the least inhibited (47\%) by 2 MIC of LEV (Table 3). VIT-C at $80 \mathrm{mg} / \mathrm{ml}$ showed the highest inhibitory effect (79\%) on Pseudomonas sp. 133 and the lowest effect (27\%) on
Klebsiella sp. 58. Similarly pattern was observed when VIT-C was used at $100 \mathrm{mg} / \mathrm{ml}$ (Table 3). Again, similar pattern of inhibitory effect on the mature biofilm pre-formed was observed regardless the degree of sensitivity or resistance of the tested uropathogens. A combination of LEV/VIT-C (2 MIC/100 mg/ml) had the highest inhibitory effect on both E. coli 27 (resistant isolate) and Pseudomonas 86 (sensitive isolate). Still both uropathogens were least inhibited when LEV was 
Citation: El-Gebaly E, Essam T, Hashem S, El-Baky RA (2012) Effect of Levofloxacin and Vitamin C on Bacterial Adherence and Preformed Biofilm on Urethral Catheter Surfaces. J Microb Biochem Technol 4: 131-136. doi:10.4172/1948-5948.1000083

\begin{tabular}{|c|c|c|c|c|c|c|c|c|c|}
\hline \multirow[b]{2}{*}{ Uropathogen } & \multicolumn{3}{|c|}{ Levofloxacin } & \multicolumn{3}{|c|}{ Vitamin-C } & \multicolumn{3}{|c|}{ Levofloxacin/Vitamin-C } \\
\hline & $\begin{array}{l}\text { Conc } \\
\mu \mathrm{g} / \mathrm{ml}\end{array}$ & $\begin{array}{c}\text { V.C } \\
\text { cfu× } 10^{3}\end{array}$ & Reduction \% & $\begin{array}{l}\text { Conc. } \\
\mathrm{mg} / \mathrm{ml}\end{array}$ & $\begin{array}{c}\text { V.C } \\
\text { cfu× } 10^{3}\end{array}$ & Reduction \% & $\begin{array}{c}\text { Conc. } \\
(\mu \mathrm{g} / \mathrm{ml}) /(\mathrm{mg} / \\
\mathrm{ml})\end{array}$ & $\begin{array}{c}\text { V.C } \\
\text { cfu× } \times 10^{3}\end{array}$ & Reduction \% \\
\hline \multirow{3}{*}{$\begin{array}{l}\text { E. coli } \\
(27)\end{array}$} & CTR & $130 \pm 10.4$ & & & $130 \pm 10.4$ & & & $130 \pm 10.4$ & \\
\hline & $128^{\mathrm{a}}$ & $80 \pm 1.1^{*}$ & 39 & 80 & $64 \pm 1^{*}$ & 51 & $128 / 80^{c}$ & $25 \pm 1.5^{*}$ & 81 \\
\hline & $256^{\mathrm{b}}$ & $39 \pm 4.5^{*}$ & 70 & 100 & $24 \pm 1.3^{*}$ & 82 & $256 / 100^{d}$ & $5 \pm 0.57^{*}$ & 96 \\
\hline \multirow{3}{*}{ (98) } & CTR & $60 \pm 5$ & & & $60 \pm 5$ & & & $60 \pm 5$ & \\
\hline & $4^{a}$ & $33 \pm 1.2^{*}$ & 45 & 80 & $50 \pm 1.1^{*}$ & 60 & $4 / 80^{c}$ & $18 \pm 0.64^{*}$ & 70 \\
\hline & $8^{\mathrm{b}}$ & $16 \pm 0.68^{*}$ & 74 & 100 & $10 \pm 0.9^{*}$ & 85 & $8 / 100^{d}$ & $2.4 \pm 0.3^{*}$ & 96 \\
\hline & & & & & & & & & \\
\hline \multirow{3}{*}{ Klebsiella sp. (56) } & CTR & $52 \pm 1.1$ & & & $52 \pm 1.1$ & & & $52 \pm 1.1$ & \\
\hline & $4^{a}$ & $16 \pm 0.64^{*}$ & 40 & 80 & $18 \pm 0.79^{*}$ & 65 & $4 / 80^{\circ}$ & $13 \pm 0.9^{*}$ & 75 \\
\hline & $8^{\mathrm{b}}$ & $4.2 \pm 0.45^{*}$ & 65 & 100 & $16 \pm 1^{*}$ & 70 & $8 / 100^{d}$ & $1 \pm 0.25^{*}$ & 95 \\
\hline \multirow{3}{*}{ (58) } & CTR & $60 \pm 1$ & & & $60 \pm 1$ & & & $60 \pm 1$ & \\
\hline & $2^{\mathrm{a}}$ & $48 \pm 1.1^{*}$ & 20 & 80 & $44 \pm 0.7^{*}$ & 27 & $2 / 80^{\circ}$ & $40 \pm 1^{*}$ & 33 \\
\hline & $4^{b}$ & $32 \pm 0.57^{*}$ & 47 & 100 & $39 \pm 0.57^{*}$ & 35 & $4 / 100^{d}$ & $10 \pm 0.57^{*}$ & 83 \\
\hline & & & & & & & & & \\
\hline \multirow{3}{*}{ Citrobacter sp. (22) } & CTR & $180 \pm 5$ & & & $180 \pm 5$ & & & $180 \pm 5$ & \\
\hline & $2^{a}$ & $67 \pm 1.5^{*}$ & 63 & 80 & $72 \pm 0.76^{*}$ & 60 & $2 / 80^{\circ}$ & $45 \pm 1^{*}$ & 75 \\
\hline & $4^{b}$ & $43 \pm 0.57^{*}$ & 76 & 100 & $27 \pm 0.57^{*}$ & 85 & $4 / 100^{d}$ & $7.2 \pm 0.4^{*}$ & 96 \\
\hline \multirow{3}{*}{ (77) } & CTR & $150 \pm 5$ & & & $150 \pm 5$ & & & $150 \pm 5$ & \\
\hline & $64^{a}$ & $75 \pm 1^{*}$ & 50 & 80 & $72 \pm 1.1^{*}$ & 52 & $64 / 80^{\circ}$ & $63 \pm 1.2^{*}$ & 58 \\
\hline & $128^{b}$ & $45 \pm 1.5^{*}$ & 70 & 100 & $53 \pm 1.5^{*}$ & 65 & $128 / 100^{d}$ & $15 \pm 1^{*}$ & 90 \\
\hline & & & & & & & & & \\
\hline \multirow{3}{*}{$\begin{array}{l}\text { Enterobacter sp. } \\
\qquad(29)\end{array}$} & CTR & $140 \pm 1.1$ & & & $140 \pm 1.1$ & & & $140 \pm 1.1$ & \\
\hline & $64^{a}$ & $90 \pm 5^{*}$ & 36 & 80 & $80 \pm 5^{*}$ & 43 & $64 / 80^{\circ}$ & $55 \pm 1^{*}$ & 61 \\
\hline & $128^{b}$ & $16 \pm 0.76^{*}$ & 89 & 100 & $42 \pm 1.1^{*}$ & 70 & $128 / 100^{d}$ & $0.5 \pm 0.15^{\star}$ & 99.6 \\
\hline \multirow{3}{*}{ (94) } & CTR & $48 \pm 1$ & & & $48 \pm 1$ & & & $48 \pm 1$ & \\
\hline & $2^{a}$ & $20 \pm 1^{*}$ & 59 & 80 & $16 \pm 0.76^{*}$ & 66.7 & $2 / 80^{\circ}$ & $14 \pm 0.76^{*}$ & 70 \\
\hline & $4^{b}$ & $10 \pm 0.76^{*}$ & 79 & 100 & $2 \pm 0.28^{*}$ & 82 & $4 / 100^{d}$ & $7.6 \pm 0.36^{*}$ & 96.5 \\
\hline & & & & & & & & & \\
\hline \multirow{3}{*}{$\begin{array}{l}\text { Proteus sp. } \\
\text { (102) }\end{array}$} & CTR & $140 \pm 5$ & & & $140 \pm 5$ & & & $140 \pm 5$ & \\
\hline & $16^{a}$ & $62 \pm 1^{*}$ & 65 & 80 & $63 \pm 1.5^{*}$ & 55 & $16 / 80^{c}$ & $42 \pm 1^{*}$ & 70 \\
\hline & $32^{b}$ & $84 \pm 1.3^{*}$ & 72 & 100 & $53 \pm 1.5^{*}$ & 62 & $32 / 100^{d}$ & $21 \pm 1^{*}$ & 85 \\
\hline \multirow{3}{*}{ (131) } & CTR & $50 \pm 1$ & & & $50 \pm 1$ & & & $50 \pm 1$ & \\
\hline & $128^{a}$ & $20 \pm 1.1^{*}$ & 60 & 80 & $28 \pm 1^{*}$ & 45 & $128 / 80^{c}$ & $18 \pm 0.28^{*}$ & 65 \\
\hline & $256^{\mathrm{b}}$ & $15 \pm 0.76^{*}$ & 70 & 100 & $18 \pm 1^{*}$ & 65 & $256 / 100^{d}$ & $6.5 \pm 0.5^{*}$ & 87 \\
\hline \multirow{3}{*}{$\begin{array}{c}\text { Pseudomonas sp. } \\
\text { (86) }\end{array}$} & CTR & $60 \pm 1.2$ & & & $60 \pm 1.2$ & & & $60 \pm 1.2$ & \\
\hline & $2^{a}$ & $37.5 \pm 0.8^{*}$ & 37.5 & 80 & $28.6 \pm 0.9^{*}$ & 55 & $2 / 80^{c}$ & $21.6 \pm 0.87^{*}$ & 64 \\
\hline & $4^{b}$ & $12 \pm 0.76^{*}$ & 80 & 100 & $12.8 \pm 0.7^{*}$ & 79 & $4 / 100^{d}$ & $3.9 \pm 0.45^{\star}$ & 93.5 \\
\hline \multirow{3}{*}{ (133) } & CTR & $76 \pm 0.76$ & & & $76 \pm 0.76$ & & & $76 \pm 0.76$ & \\
\hline & $16^{a}$ & $28 \pm 1^{*}$ & 63 & 80 & $16 \pm 0.76^{*}$ & 79 & $16 / 80^{c}$ & $14 \pm 0.76^{*}$ & 82 \\
\hline & $32^{\mathrm{b}}$ & $6.9 \pm 0.49^{*}$ & 91 & 100 & $8.4 \pm 0.3^{*}$ & 89 & $32 / 100^{d}$ & $1.5 \pm 0.28^{*}$ & 98 \\
\hline \multicolumn{10}{|c|}{$\begin{array}{l}\text { a) The used concentration was equal to MIC of levofloxacin alone. } \\
\text { b) The used concentration was equal to } 2 \mathrm{MIC} \text { of levofloxacin alone. } \\
\text { c) The used concentration was equal to MIC of levofloxacin and } 80 \mathrm{mg} \text { vitamin } \mathrm{C} / \mathrm{ml} \text {. } \\
\text { d) The used concentration was equal to } 2 \mathrm{MIC} \text { of levofloxacin and } 100 \mathrm{mg} \text { vitamin } \mathrm{C} / \mathrm{ml} \text {. } \\
\text { (V.C) means the viable cell counts on the surface of urethral catheters. } \\
\text { (CTR) means the control without drug. } \\
{ }^{*} \text { P<0.05: Significant value, compared to controls. }\end{array}$} \\
\hline
\end{tabular}

Table 3: Effects of levofloxacin, vitamin-C alone and in combination on the preformed biofilm on the catheter surfaces.

used alone at MIC (Figure 2). The effect of levofloxacin and vitamin-C either individually used or in combination was ascertained by SEM where at $0.5 \mathrm{MIC}$ or VIT-C $(100 \mathrm{mg} / \mathrm{ml})$ a decrease in the number of adherent cells of all tested uropathogens was observed (Figure 3). This was always accompanied with morphological changes where the cells were elongated with disorganized membrane especially when vitamin-C was used (Figure 3B and 3D). A distinct decrease in number accompanied with scattering, swallowing and malformation of the adherent cells were observed upon combination of both levofloxacin and vitamin-C (Figure 3F).

\section{Discussion}

Static adherence assay demonstrated that the effect of levofloxacin and vitamin-C on biofilm inhibition was concentration dependent. 
Citation: El-Gebaly E, Essam T, Hashem S, El-Baky RA (2012) Effect of Levofloxacin and Vitamin C on Bacterial Adherence and Preformed Biofilm on Urethral Catheter Surfaces. J Microb Biochem Technol 4: 131-136. doi:10.4172/1948-5948.1000083

Levofloxacin at sub-MIC concentrations (0.25 MIC and $0.5 \mathrm{MIC})$ reduced biofilm synthesis by $40-96 \%$. The inhibitory effect of sub-MIC of levofloxacin against biofilm formation was also similarly reported by Drago et al. [16] who have recorded a significant inhibition of bacterial adherence to uroepithelial cells when both ciprofloxacin and levofloxacin were used at sub-inhibitory concentrations. Similarly, it was reported that the presence of sub-inhibitory concentrations $(0.5$, 0.25 , or $0.125 \mathrm{MIC}$ ) of all the tested antimicrobial agents significantly reduced the biofilm formation and adherence of $P$. aeruginosa and $S$. aureus to plastic surfaces [17].

Both levofloxacin and vitamin-C had similar inhibitory effect on the preformed biofilm. However, higher concentrations of levofloxacin were needed for reduction of pre-formed biofilm than those required in case of initial microbial adherence. Levofloxacin at concentrations of MIC and 2MIC reduced the viable cell counts of the mature biofilm by $36-95 \%$ of the controls. Similarly, it was demonstrated that a high concentration of ciprofloxacin (another member of quinolones antibiotics) was necessary for significant reduction of a pre-formed biofilm, although the drug could reduce the adhesion and survival of the Pseudomonas aeruginosa at subinhibitory concentrations $[15,18]$. Vitamin-C was tested for its ability to inhibit biofilm formation and there was up to $92 \%$ reduction in the number of viable adherent bacteria on catheters treated with 80 and $100 \mathrm{mg}$ vitamin- $\mathrm{C} \mathrm{m} \mathrm{ml}^{-1}$. Also a reduction of viable counts of the mature biofilm by up to $89 \%$ was

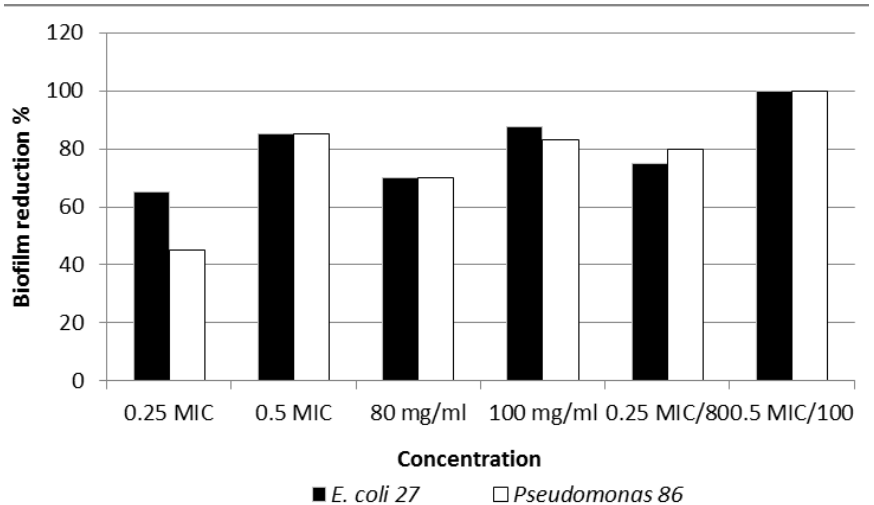

Figure 1: Effect of levofloxacin and vitamin-C at different concentrations on biofilm production on urethral catheter surfaces by $=E$. coli 27 and $\square$ Pseudomonas 86.

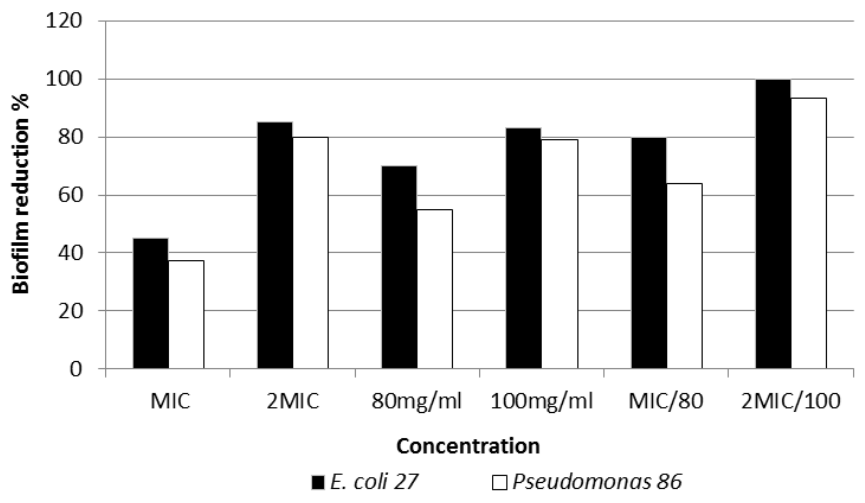

Figure 2: Effect of levofloxacin and vitamin-C at different concentrations on the preformed biofilm on urethral catheter surfaces by $\square$. coli 27 and $\square$ Pseudomonas 86 .
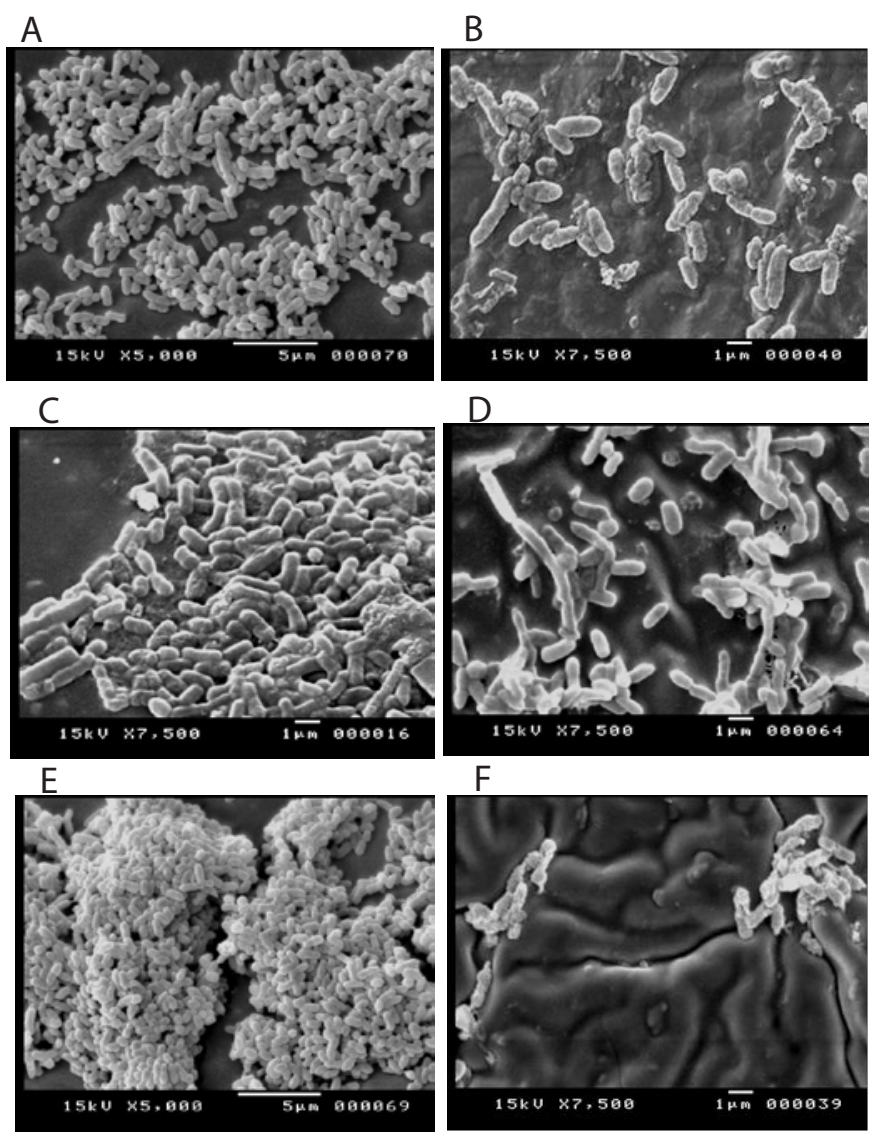

$\mathrm{F}$

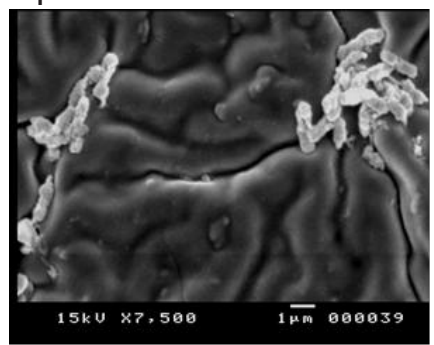

Figure 3: Scanning electron micrographs showing growth and adherence of different uropathogens on the surface of equally sized urethral catheters segments incubated for $24 \mathrm{~h}$.

(A) Initial growth and adherence of Citrobacter sp. without any treatment.

(B) Growth and adherence of Citrobacter sp. treated with levofloxacin at 0.5 MIC.

(C) Initial growth and adherence of $E$. coli without any treatment.

(D) Growth and adherence of E. coli treated with vitamin-C at $100 \mathrm{mg} / \mathrm{ml}$.

(E) Initial growth and adherence of Proteus sp. without any treatment.

(F) Growth and adherence of Proteus sp. treated with combination of LEV/VIT-C at $0.5 \mathrm{MIC} / 100 \mathrm{mg} / \mathrm{ml}$.

observed. Ascorbic acid (vitamin-C) is a naturally occurring furanone [19], and could be exploited to control quorum sensing in bacteria [20]. Therefore, the inhibitory effect of vitamin-C might be due to its anti-quorum sensing activity where ascorbic acid was reported to have competitive inhibition with autoinducer-2 (AI-2) [20]. Previously, Habash et al. [11] demonstrated that vitamin-C supplementation can provide a degree of protection against adhesion by some uropathogens and colonization of biomaterials utilized within the urinary tract and this agrees with the results reported in the present study. Again, it has been reported that there was a steady decrease in viable cell counts with increasing vitamin-C concentrations that might have impacted growth [20]. Combination of levofloxacin with vitamin-C (LEV/VIT-C) increased the inhibitory effects of the initial microbial adherence to the catheter surface up to $100 \%$ with almost complete eradication of the pre-formed biofilm. The enhancement of the inhibitory effect of LEV/VIT-C combinations could be explained by the fact that reducing $\mathrm{pH}$ of the medium by vitamin- $\mathrm{C}$ addition could increase the activity of levofloxacin according to what have been reported by [17].

A similar pattern was reported by El-Feky et al. [21]; where 
Citation: El-Gebaly E, Essam T, Hashem S, El-Baky RA (2012) Effect of Levofloxacin and Vitamin C on Bacterial Adherence and Preformed Biofilm on Urethral Catheter Surfaces. J Microb Biochem Technol 4: 131-136. doi:10.4172/1948-5948.1000083

ciprofloxacin and $\mathrm{N}$-acetylcysteine combinations had better inhibitory effect on both biofilm formation and pre-formed biofilm than that recorded for individually used drugs. However, levofloxacin is more advantageous than ciprofloxacin as lower concentrations of it (subMIC) showed almost the same inhibitory effect to ciprofloxacin at higher concentrations. Levofloxacin was also reported to have almost double the renal excretion rate of ciprofloxacin and this makes it an ideal agent for urinary tract infections (UTIs) [22]. Similarly, vitamin-C is more preferable than $\mathrm{N}$-acetylcystiene due to both terms; safety and cost. In conclusion, these results showed the tremendous increase in inhibitory effect of the biofilm formation upon combination of both levofloxacin and vitamin- $\mathrm{C}$ and this represents a promising approach for prevention and treatment of biofilm associated infections.

\section{References}

1. Barford JMT, Coates ARM (2009) The pathogenesis of catheter-associated urinary tract infection. J Infect Prev 10: 50-56.

2. Lee MK, Chiu CS, Chow VC, Lam RK, Lai RW (2007) Prevalence of hospital infection and antibiotic use at a university medical center in Hong Kong. J Hosp Infect 65: 341-347.

3. Tambyah PA, Knasinski V, Maki DG (2002) The direct costs of nosocomial catheter-associated urinary tract infection in the era of managed care. Infect Control Hosp Epidemiol 23: 27-31.

4. Burton E, Yakandawala N, LoVetri K, Madhyastha MS (2007) A microplate spectrofluorometric assay for bacterial biofilms. J Ind Microbiol Biotechnol 34: $1-4$.

5. Stoodley P, Sauer K, Davies DG, Costerton JW (2002) Biofilms as complex differentiated communities. Annu Rev Microbiol 56: 187-209.

6. Mohamed JA, Huang DB (2007) Biofilm formation by enterococci. J Med Microbiol 56: 1581-1588.

7. Tenke P, Kovacs B, Jäckel M, Nagy E (2006) The role of biofilm infection in urology. World J Urol 24: 13-20.

8. Norrby SR, Petermann W, Willcox PA, Vetter N, Salewski E (1998) A comparative study of levofloxacin and ceftriaxone in the treatment of hospitalized patients with pneumonia. Scand J Infect Dis 30: 397-404.

9. Ishida H, Ishida Y, Kurosaka Y, Otani T, Sato K, et al. (1998) In vitro and in vivo activities of levofloxacin against biofilm-producing Pseudomonas aeruginosa.
Antimicrob Agents Chemother 42: 1641-1645.

10. Carlsson S, Weitzberg E, Wiklund P, Lundberg JO (2005) Intravesical nitric oxide delivery for prevention of catheter-associated urinary tract infections. Antimicrob Agents Chemother 49: 2352-2355.

11. Habash MB, Van der Mei HC, Busscher HJ, Reid G (1999) The effect of water ascorbic acid, and cranberry derived supplementation on human urine and uropathogen adhesion to silicone rubber. Can J Microbiol 45: 691-694.

12. Olofsson AC, Hermansson M, Elwing H (2003) N-acetyl-L-cysteine affects growth, extracellular polysaccharide production, and bacterial biofilm formation on solid surfaces. Appl Environ Microbiol 69: 4814-4822.

13. Tamer E, Amin MA, El Tayeb O, Mattiasson B, Guieysse B (2010) Characterization of highly resistant phenol degrading strain isolated from industrial wastewater treatment plant. J Hazardous materials 173: 783-788.

14. Clinical and laboratory standards institutes (2009) Performance standards for antimicrobial disk susceptibility tests; Approved standard. (10thedn), M02-A10, Wayne, PA.

15. Reid G, Sharma S, Advikolanu K, Tieszer C, Martin RA, et al. (1994) Effects of ciprofloxacin, norfloxacin, and ofloxacin on in vitro adhesion and survival of Pseudomonas aeruginosa AK1 on urinary catheters. Antimicrob Agents Chemother 38: 1490-1495.

16. Drago L, De Vecchi E, Mombelli B, Nicola L, Valli M, et al. (2001) Activity of levofloxacin and ciprofloxacin against urinary pathogens. J Antimicrob Chemother 48: 37-45.

17. Yassein MAM (2004) Studies on the prevention of adherence of biofilm associated bacteria to human cells and implanted medical devices.

18. Yassien M, Khardori N, Ahmedy A, Toama M (1995) Modulation of biofilms of Pseudomonas aeruginosa by quinolones. Antimicrob Agents Chemother 39 2262-2268.

19. Colin Slaughter J (1999) The naturally occurring furanones: formation and function from pheromone to food. Biol Rev Camb Philos Soc 74: 259-276.

20. Novak JS, Fratamico PM (2004) Evaluation of ascorbic acid as a quorumsensing analogue to control growth, sporulation, and enterotoxin production in Clostridium perfringens. J Food Sci 69.

21. El-Feky MA, El-Rehewy MS, Hassan MA, Abolella HA, Abd El-Baky RM, et al. (2009) Effect of ciprofloxacin and $\mathrm{N}$-acetylcysteine on bacterial adherence and biofilm formation on ureteral stent surfaces. Pol J Microbiol 58: 261-267.

22. Concia E, Azzini AM (2008) Levofloxacin for the treatment of urosepsis 19-24. 